LAWRENCE LIVERMORE NAT IO N A L LABORATORY

\section{Air-Flow Simulation in Realistic Models of the Trachea}

T. Deschamps, P. Schwartz, D. Trebotich

December 10, 2004

Engineering in Medicine and Biology Society Conference San Francisco, CA, United States

September 1, 2004 through September 5, 2004 
This document was prepared as an account of work sponsored by an agency of the United States Government. Neither the United States Government nor the University of California nor any of their employees, makes any warranty, express or implied, or assumes any legal liability or responsibility for the accuracy, completeness, or usefulness of any information, apparatus, product, or process disclosed, or represents that its use would not infringe privately owned rights. Reference herein to any specific commercial product, process, or service by trade name, trademark, manufacturer, or otherwise, does not necessarily constitute or imply its endorsement, recommendation, or favoring by the United States Government or the University of California. The views and opinions of authors expressed herein do not necessarily state or reflect those of the United States Government or the University of California, and shall not be used for advertising or product endorsement purposes. 


\title{
Air-flow simulation in realistic models of the trachea
}

\author{
T. Deschamps ${ }^{1}$, P. Schwartz ${ }^{2}$, D. Trebotich $^{3}$ \\ ${ }^{1}$ Mathematics Department, Lawrence Berkeley National Laboratory, USA \\ 2 Applied Numerical Algorithms Group, Lawrence Berkeley National Laboratory, USA \\ ${ }^{3}$ Center for Applied Scientific Computing, Lawrence Livermore National Laboratory, USA
}

\begin{abstract}
In this article we present preliminary results from a new technique for flow simulation in realistic anatomical airways. The airways are extracted by means of Level-Sets methods that accurately model the complex and varying surfaces of anatomical objects. The surfaces obtained are defined at the sub-pixel level where they intersect the Cartesian grid of the image domain. It is therefore straightforward to construct embedded boundary representations of these objects on the same grid, for which recent work has enabled discretization of the NavierStokes equations for incompressible fluids. While most classical techniques require construction of a structured mesh that approximates the surface in order to extrapolate a 3D finite-element griding of the whole volume, our method directly simulates the air-flow inside the extracted surface without losing any complicated details and without building additional grids.
\end{abstract}

Index Terms-Air-Flow, Segmentation, Level-Sets, FastMarching, Navier-Stokes equations, Projection Methods, Embedded Boundary Methods

\section{INTRODUCTION}

Computational Fluid Dynamics (CFD) simulations of complex flows in narrow passages of the airways can provide clinicians with information needed to evaluate how pathologies form, how they evolve, and ultimately how they are effectively treated. In order to do this, accurate methods are required to build the anatomical models, and CFD codes must be available that can run on anatomical models such as a trachea (with a tracheotomy) as in figure 1 .

However, methods of surface extraction are limited in their ability to recover the geometry of complex objects like tubular branching structures in real time. Moreover, it is necessary to convert the surface extracted by image processing techniques into a computational domain appropriate for the CFD solver, involving the construction of a mesh on the surface and in the 3D

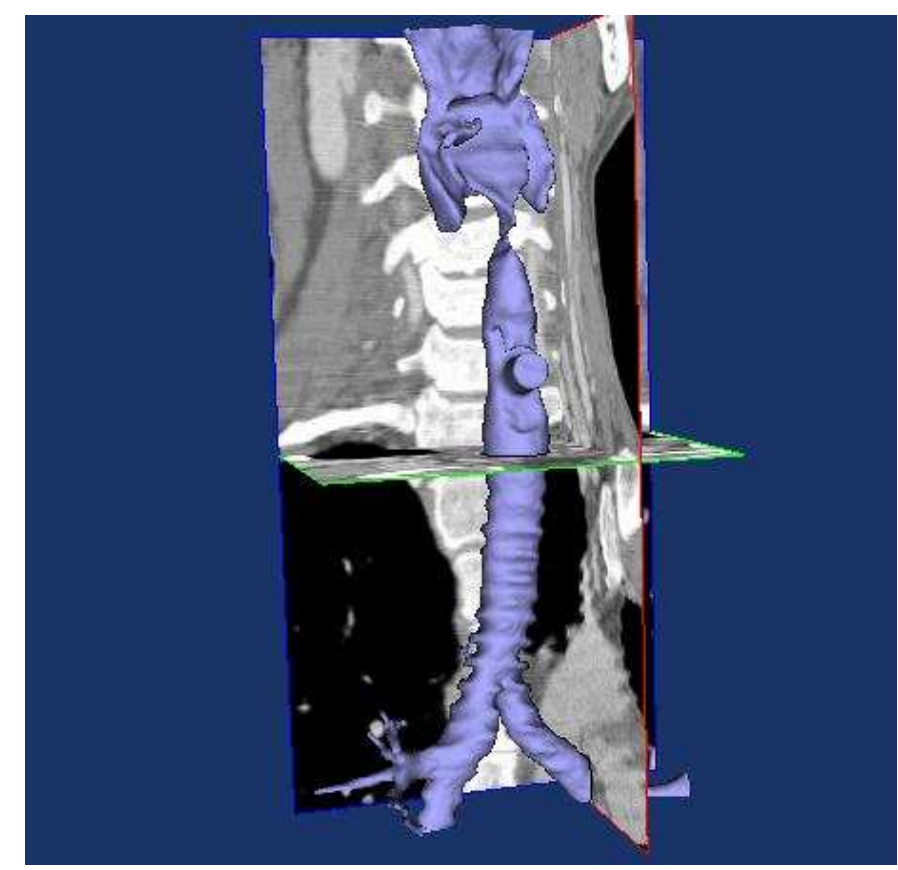

Fig. 1. Surface Rendering of a Trachea from a 3D CT dataset

domain surrounding it. Valuable information can be lost during this construction due to limitations of the CFD solvers with respect to the properties of the surface mesh. It is often the case, for example, that the surface needs to be smoothed in order to build a finite-element mesh necessary to some CFD codes. Failing that, irregular surfaces must be approximated by a large number of small mesh elements, pushing the limits of computer memory. The result in this and other cases is a compromise in the level of accuracy of the surface extraction method.

Some recent techniques of image processing enable an approximation of the surface of any tubular object in the patient body to be built using CT or MR images of the patient enhanced with contrast product. This work is based on an accurate surface extraction with front 
propagation techniques based on the Fast-Marching and Level-Sets methods [1].

In addition, recent techniques in CFD make easy use of these approximate surfaces in the generation of computational grids: a higher-order projection method has been designed on Chombo, a software framework for applied partial differential equation (PDE) solvers on irregular domains.

The purpose of this work, therefore, is to transform the result of an accurate surface extraction method detailed in section II into a CFD mesh detailed in section III with no loss of information. Using a high-resolution CFD code, we accurately compute measures of fluid velocity and pressure in realistic geometries, presented in section IV.

\section{Surface Extraction}

Fast-Marching and Level-Sets methods are numerical techniques which can follow the evolution of contours and surfaces that can develop sharp corners, break apart, and merge together, and are particularly useful for shape recovery of complex geometries like anatomical objects [2].

Rather than tracking the movement of a given contour $C$ moving with speed $F$ in its normal direction $\vec{n}$ according to the evolution equation $\frac{\partial C}{\partial t}=F \vec{n}$, we consider the signed distance function $\phi$ to $C$ and track its motion with the evolution equation

$$
\begin{aligned}
\frac{\partial \phi}{\partial t} & =F|\nabla \phi|, \\
\text { with } F & =k_{I}(1-\epsilon \kappa)-\nabla k_{I} \cdot \vec{n}, \\
\text { and } k_{I}(\mathbf{x}) & =e^{-\|\nabla I \mathbf{x}\|}
\end{aligned}
$$

This is the Level-Sets method [1]. Therefore since the contour is moved implicitly by the evolution of the signed distance function, the resulting surface extraction exhibits good properties including a total adaptability to complex topologies like branching structures and a sub-pixel accuracy of the surface thanks to the implicit formulation of the sign distance function. An example is shown in figure 2 When the speed $F$ is strictly positive and a function of the image only, equation 1 resolves in the stationary case $F|\nabla T|=1$, with $F(\mathbf{x})=$ $k_{I}(\mathbf{x})$ defined in eq (1) where $T$ is the crossing time of the propagating front. Since Level-Sets are rather slow, initialization of the segmentation can be done with the Fast-Marching method [1] that solves Eikonal equation in $O(n \log (n))$ (where $n$ is the number of points in the image). When coupled [3], these numerical techniques can achieve the highest level of accuracy in a very short time, compared to other segmentation techniques like classical active contours that fail to capture complex shapes. These algorithms have been validated in numerous cases [4], and can be specifically optimized to extract long and thin tubular objects [5].

\section{BUILDING THE CFD GRID}

In order to simulate the air-flow inside the segmented trachea, many methods rely on a finite element griding of the 3D domain that is based on re-meshing appropriately the surface extracted. Re-meshing often discards a lot of the surface details because it is done with approximation of the surface (like NURBS) and involves a lot of user-interaction. Other methods that want to keep all the details end up with quantity of mesh elements (like triangles) that are challenging both for computers' memory and commercial CFD softwares.

The embedded boundary method is an approach in which regular cells on a Cartesian grid are cut by physical boundaries and/or interfaces (see Figure 3). Higherorder stencils are constructed near boundaries where irregular, or "cut", cells occur while discretizations with known accuracy and stability requirements are employed on the interior regular cells [6], [7].

The geometric facts required for numerical methods (normals, centroids, face areas, and so forth) can each be realized as integrals of monomials over the volume-offluid. There is in general more than one such integral for each geometric datum. Hence the set of all such integrals constitutes an overdetermined system for the geometry. We apply Least Squares to this over-determined system, which finesses some difficult aspects of computational geometry. Furthermore, by using the divergence theorem we are able to replace volume integrals over irregular regions with trivial computations of one-dimensional integrals over line-segments.

Given a level set description of the EB as above, grid generation proceeds as an automated process taking a few minutes (see figure 3 ).

\section{Air-Flow Simulation}

We make use of the Chombo software libraries [8] which provide a framework for data management, numerical operators and linear solvers needed in an EB formulation. We model air-flow with the incompressible 

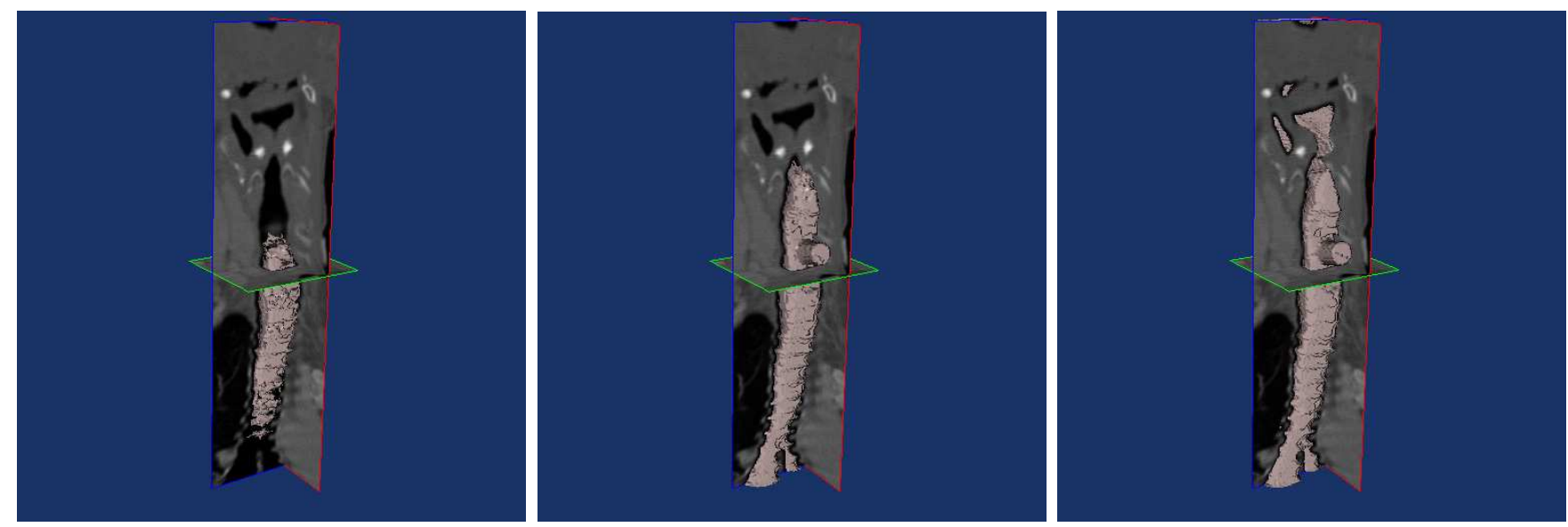

Fig. 2. Trachea segmentation with Level-Sets: the surface intersects three orthogonal planes mapped with the gray-level values of the 3D CT dataset.
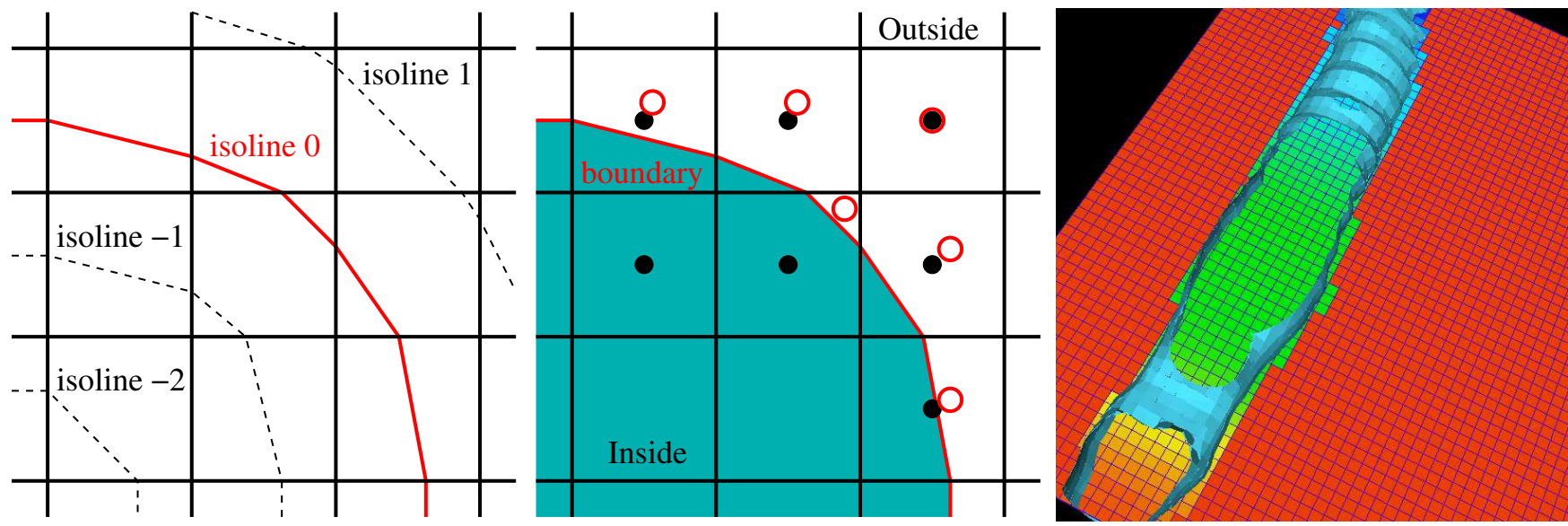

Fig. 3. From Level-Sets to Embedded Boundary - Left image: the distance function on the Cartesian grid - Middle image: the different cells, their centers (black disks) and centroids (empty disks) - Right Image: the Surface and the underlying Cartesian grid for the trachea example.

Navier-Stokes equations using a projection method [9], [10]:

$$
\rho u_{t}+\rho(u \cdot \nabla) u=-\nabla p+\mu_{s} \Delta u, \text { and } \nabla \cdot u=0 .
$$

We present in figure 4 results for the flow simulated in the trachea. Air velocity and pressure are computed with the flow solver and visualized on a desktop workstation. Pressure drops (brighter areas) and complex flow patterns are observed.

\section{CONCLUSION}

Our approach to computing flows in realistic geometries does not modify the object geometry from the imaging, and uses a fast, automated process for generating an appropriate finite volume grid. We have a direct construction of the embedded boundary on the Cartesian grid, and thus no loss of information nor accuracy. The final flow simulation results are obtained for the realistic geometry that has been extracted by the segmentation method. In future work we will improve the resolution of the flow solver using adaptive mesh refinement.

\section{ACKNOWLEDGMENT}

We would like to recognize Ravi Malladi, Math group LBNL and Phil Colella, head of the Applied Numerical Algorithms Group, LBNL for their help and support in our work. This work was performed under the auspices of the U.S. Department of Energy by the University of California, Lawrence Livermore National Laboratory under contract No. W-7405-Eng-48. 

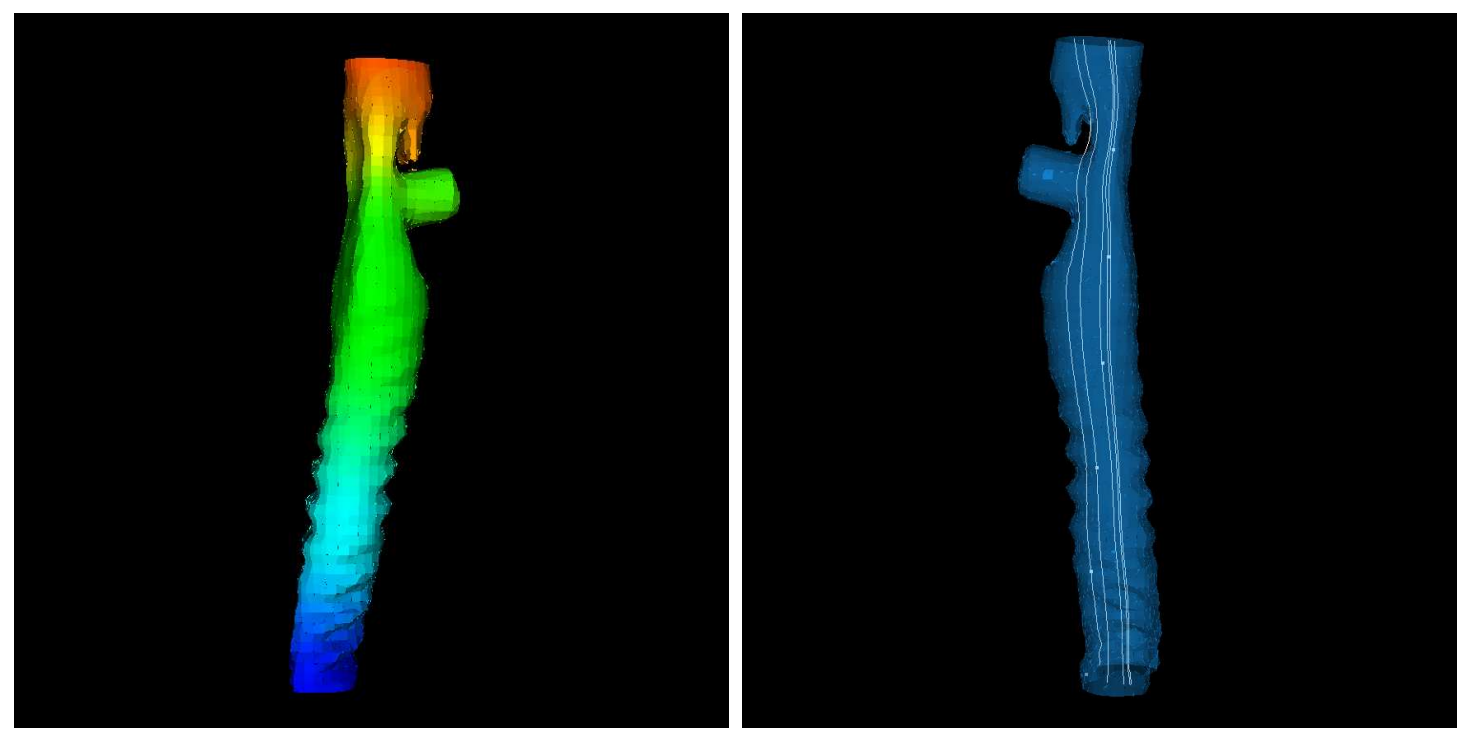

Fig. 4. Pressure and flow streamlines from Air-Flow simulation inside the trachea

\section{REFERENCES}

[1] J.A. Sethian, Level set methods: Evolving Interfaces in Geometry, Fluid Mechanics, Computer Vision and Materials Sciences, Cambridge University Press, UC. Berkeley, 1999.

[2] R. Malladi, J.A. Sethian, and B.C. Vemuri, "Shape modelling with front propagation: A level set approach," IEEE Trans. Pattern Analysis And Machine Intelligence, vol. 17, no. 2, pp. 158-175, Feb. 1995.

[3] R. Malladi and J.A. Sethian, "A real-time algorithm for medical shape recovery," Jan. 1998, pp. 304-310.

[4] M. Hernandeza, R. Barrenab, G. Hernandezb, G. Sapiroc, and A.F. Frangi, "Pre-clinical evaluation of implicit deformable models for three-dimensional segmentation of brain aneurysms in cta," in SPIE Medical Imaging, 2003, vol. 5032, pp. 12641274.

[5] T. Deschamps and L.D. Cohen, "Fast surface and tree structure extraction of vascular objects in 3d medical images," in Intl. Conf. on Curves and Surfaces, Saint-Malo, France, June 2002.

[6] H. Johansen and P. Colella, "A cartesian grid embedded boundary method for poisson's equation on irregular domains," J. Comput. Phys., vol. 147, no. 2, pp. 60-85, dec 1998.

[7] P. McCorquodale, P. Colella, and H. Johansen, "A cartesian grid embedded boundary method for the heat equation on irregular domains," J. Comput. Phys., vol. 173, pp. 620-635, 2001.

[8] P. Colella, D.T. Graves, T.J. Ligocki, D.F. Martin, D. Modiano, D.B. Serafini, and B. Van Straalen, "Chombo software package for amr applications," 2000.

[9] A. Chorin, "Numerical solutions of the navier-stokes equations," Math. Comp., vol. 22, 1968.

[10] J.B. Bell, P. Colella, and H.M. Glaz, "A second-order projection method for the incompressible navier-stokes equations," $J$. Comput. Phys., vol. 85, pp. 257-283, 1989. 